\title{
Piezoelectric properties of zinc oxide/iron oxide filled polyvinylidene fluoride nanocomposite fibers
}

\author{
Abdulrahman Mohmmed AIAhzm ${ }^{1}$, Maan Omar Alejli ${ }^{1}$, Deepalekshmi Ponnamma ${ }^{2, *}$ (1), \\ Yara Elgawady ${ }^{2}$, and Mariam Al Ali Al-Maadeed ${ }^{2}$ \\ ${ }^{1}$ Department of Chemistry, College of Arts and Science, Qatar University, P O Box 2713, Doha, Qatar \\ ${ }^{2}$ Center for Advanced Materials, Qatar University, P. O. Box 2713, Doha, Qatar
}

Received: 21 December 2020

Accepted: 18 April 2021

Published online:

3 June 2021

(C) The Author(s) 2021

\begin{abstract}
Piezoelectric nanogenerators (PENG) with flexible and simple design have pronounced significance in fabricating sustainable devices for self-powering electronics. This study demonstrates the fabrication of electrospun nanocomposite fibers from polyvinylidene fluoride (PVDF) filled zinc oxide $(\mathrm{ZnO}) /$ iron oxide $(\mathrm{FeO})$ nanomaterials. The nanocomposite fiber based flexible PENG shows piezoelectric output voltage of $5.9 \mathrm{~V}$ when $3 \mathrm{wt} \%$ of $\mathrm{ZnO} / \mathrm{FeO}$ hybrid nanomaterial is introduced, which is 29.5 times higher than the neat PVDF. No apparent decline in output voltage is observed for almost $2000 \mathrm{~s}$ attributed to the outstanding durability. This higher piezoelectric output performance is correlated with the $\beta$-phase transformation studies from the Fourier transformation infrared spectroscopy and the crystallinity studies from the differential scanning calorimetry. Both these studies show respective enhancement of 3.79 and $2.16 \%$ in the $\beta$-phase crystallinity values of PVDF-ZnO/FeO 3 wt $\%$ composite. Higher dielectric constant value obtained for the same composite (three times higher than the neat PVDF) confirms the increased energy storage efficiency as well. Thus the proposed soft and flexible PENG is a promising mechanical energy harvester, and its good dielectric properties reveals the ability to use this material as good power sources for wearable and flexible electronic devices.
\end{abstract}

\section{Introduction}

With the growing global energy demand and the rapidly escalating environmental issues, the pursuit of innovating new and safe energy sources became more urgent [1, 2]. Rechargeable power and renewable energy sources can be considered as alternative solutions to build up a sustainable environment [3].

Address correspondence to E-mail: lekshmi_deepa@yahoo.com
Piezoelectric materials come to light in recent times provide green solutions to develop flexible, biocompatible, light weight and environmental friendly mechanical energy harvesters [4]. Such kind of devices are used as self-powering resources for electronic skin sensors, robotics and health care monitoring systems [5]. Piezoelectric nanogenerators (PENG) could harvest energy from acoustic waves 
and human activities other than mechanical vibrations to power liquid crystal displays and light emitting diodes [6-9].

Numerous ceramic materials such as $\mathrm{PbZr}_{x} \mathrm{Ti}_{1-x} \mathrm{O}_{3}$, $\mathrm{BaTiO}_{3}(\mathrm{BT}), \mathrm{SrTiO}_{3}, \mathrm{ZnSnO}_{3},(\mathrm{~K}, \mathrm{Na}) \mathrm{NbO}_{3}$ etc. $[10,11]$, inorganic semiconductors like $\mathrm{ZnO}$, In $\mathrm{N}$, GaN etc. [12], ferroelectric perovskite such as $\mathrm{BiFeO}_{3}$ [13], and polymers such as polyvinylidene fluoride (PVDF) and its copolymers (poly(vinylidenefluorideco-trifluoroethylene), polyvinylidene fluoride hexafluoropropylene (PVDF-HFP)) [14, 15] are widely reported for manufacturing PENGs. PVDF is a synthetic semi crystalline polymer, and its $\beta$-phase and $\gamma$-phases are responsible for the piezoelectric power harvesting property. These electroactive phases subsequently improve with dipole alignment processes like corona poling and with the addition of nanomaterials [16]. Electrospinning is identified as a good manufacturing technique, which causes the dipole alignment as an alternative to additional poling processes [17]. Moreover, soft and flexible fibers satisfy the lightweight and convenient designs useful for industrial applications. Various nucleating nanofillers added to polymeric medium also improve the thermal, electrical, and mechanical properties, in addition to the piezoelectricity $[17,18]$.

Metal oxide nanoparticles like $\mathrm{ZnO}, \mathrm{NiO}$ etc. can enhance the $\beta$-phase content of the PVDF based polymers and thus can increase the piezoelectric performance [19]. This is because of the cubic close packed structure of them and the associated high surface energy stabilizing the unstable thermodynamic phases. The nonnative crystal phase and the center of symmetry of these metal oxides also improves the physiochemical properties of other materials when coupled [19]. Magnetoelectric polymer composites are also reported with good piezoelectric output voltage [20]. $\mathrm{ZnO}$ is an outstanding filler with significant research interest in this regard as its polymer composites also show excellent mechanical properties, and are lower in cost, and less toxic [12]. However, all metal oxides limit their fundamental applications by the agglomeration and low percolation issues, and often various methods must be practiced prior to the composite formation to enhance the nanoparticle efficiency [5]. Dutta et al. coated $\mathrm{NiO}$ particles with $\mathrm{SiO}_{2}$, to develop a homogeneous filler distribution in the polymer medium and achieved high output voltage $(53 \mathrm{~V})$, and high power density $\left(685 \mathrm{~W} / \mathrm{m}^{3}\right)$ for the $\mathrm{NiO@SiO} 2 / \mathrm{PVDF}$ composites [5]. Our research group has practiced the additional doping of metal oxide with specific ions ( $\mathrm{Fe}, \mathrm{Co}$ and $\mathrm{Ni}$ ) to generate doped $\mathrm{ZnO}$ nanomaterials with various structural and morphology features [21-23]. Such composites, depending on the fabrication method, produced 2-12 V output voltage, even from a very small piece of composite sample.

Multifunctional materials are targeted with additional optical, electrical and thermal stability characteristics in addition to piezoelectricity to extend the applicability of such composites [24]. In piezoelectricity, Fe compounds are often used due to its magnetic and ferroelectric property, which further contribute to piezoelectric voltage generation [25]. $\mathrm{ZnO}$ heterojunctions enhance the charge separation and influence the charge transfer reactions at the interface. This variable heterojunction characteristics due to the presence of additional nanomaterials can cause positive effects in regulating the piezoelectricity [26]. Nam and coworkers reviewed the physicochemical properties and phase transformations occurring in non-native transition metal oxides such as hexagonal $\mathrm{ZnO}$ and the cubic $\mathrm{FeO}, \mathrm{CoO}$ and $\mathrm{NiO}$ [19]. The hexagonal crystal structure of $\mathrm{ZnO}$ with the tetrahedrally coordinated $\mathrm{Zn}^{2+}$ cations and $\mathrm{O}^{2-}$ anions itself is supporting its piezoelectricity, and the additional filler coupling with good stability further enhances the piezoelectric mechanism.

This work aims in fabricating $\mathrm{ZnO}-\mathrm{FeO}$ hybrid nanomaterials with good heterojunction to improve the piezoelectric properties of the electrospun PVDF composite fibers. The high stretching force during electrospinning produces self-poled piezoelectric nanofibers and the inorganic fillers mechanically and thermally improves the sample. Hybrid fillers cause good distribution within the polymer and the effect of crystallinity variations on the piezoelectric performance evaluates the efficiency and durability of the PENGs. Low cost, flexible and reliable fibers for soft robotics, and other self-powering flexible electronic devices are targeted by this study.

\section{Experimental protocol}

\subsection{Materials}

The polymer, PVDF of molecular weight 180,000 was purchased from Sigma Aldrich, along with the solvents dimethyl formamide (DMF), acetone and 
ethanol. All chemicals used to synthesize the $\mathrm{ZnO}$ and $\mathrm{FeO}$ nanomaterials such as zinc acetate $\left[\mathrm{Zn}\left(\mathrm{CH}_{3} \mathrm{COO}\right)_{2} \cdot 2 \mathrm{H}_{2} \mathrm{O}\right]$, polyethylene glycol (PEG), monoethanolamine or MEA $\left[\mathrm{C}_{2} \mathrm{H}_{7} \mathrm{NO}\right]$ and iron chloride were also commercially obtained from the Sigma Aldrich.

\subsection{Synthesis of nanomaterials}

For the synthesis of $\mathrm{ZnO}$, about $2 \mathrm{~g}$ of $\mathrm{Zn}\left(\mathrm{CH}_{3-}\right.$ $\mathrm{COO})_{2} \cdot 2 \mathrm{H}_{2} \mathrm{O}$ was dissolved in $50 \mathrm{ml}$ deionized water, by magnetically stirring the solution for $30 \mathrm{~min}$ at $750 \mathrm{rpm} .0 .5 \mathrm{~g}$ of PEG was then added to the solution with stirring for another $30 \mathrm{~min}$, followed by $3 \mathrm{ml}$ MEA. The solution was stirred again for $15 \mathrm{~min}$, transferred to a pre-heated autoclave, and treated at $140{ }^{\circ} \mathrm{C}$ for $15 \mathrm{~min}$. When the hydrothermal reaction was finished, the autoclave was allowed to cool to room temperature, and the obtained precipitate was washed using water and ethanol. The powder collected after the filtration process was calcined at 400 ${ }^{\circ} \mathrm{C}$ for $2 \mathrm{~h}$ to finish the drying process [22].

$\mathrm{FeO}$ nanopowder was synthesized by dissolving $2 \mathrm{~g} \mathrm{FeCl}_{2}$ in $50 \mathrm{ml}$ deionized water followed by the addition of PEG and MEA as in the case of $\mathrm{ZnO}$ nanomaterial, but following hot plate method by heating the solution at $80{ }^{\circ} \mathrm{C}$ for $1 \mathrm{~h}$. Washing and drying process were followed to obtain the dried nanopowder. For the preparation of hybrid $\mathrm{FeO} /$ $\mathrm{ZnO}$, hydrothermal $\mathrm{ZnO}$ synthesis was repeated in the presence of $0.2 \mathrm{~g}$ of $\mathrm{FeO}$ (dissolved in distilled water). All three nanomaterials- $\mathrm{ZnO}, \mathrm{FeO}$ and $\mathrm{FeO} /$ $\mathrm{ZnO}$ were dried and used for the synthesis of polymer nanocomposites.

\subsection{Fabrication of polymer nanocomposite fibers}

PVDF dissolved in an equimolar mixture of DMF and Acetone solvents in 18-wt\% (1.8 g PVDF in $10 \mathrm{ml}$ solvent mixture) was used for electrospinning. Solution feed rate, and needle to collector distance were maintained respectively at $1 \mathrm{ml} / \mathrm{h}$, and $12 \mathrm{~cm}$. All these parameters were optimized based on the obtained uniform and beads free fiber morphology. Defect free fibers were obtained on a rotating drum when the applied voltage exceeded $10 \mathrm{~V}$. In order to prepare the polymer nanocomposite, nanofillers such as $\mathrm{ZnO}, \mathrm{FeO}$ and $\mathrm{FeO} / \mathrm{ZnO}$ were added to the PVDF in $1 \mathrm{wt} \%$. A higher concentration of the hybrid $\mathrm{FeO} /$
$\mathrm{ZnO}$ nanomaterials was also made by dispersing 3 wt $\%$ of the filler concentration. The nanomaterial concentration was determined based on the amount of the PVDF polymer. In all cases, the nanomaterials were dispersed in the same DMF/Acetone solvent mixture by bath sonication, and later mixed with the polymer by overnight magnetic stirring process.

\subsection{Characterization techniques}

Scanning electron microscope (SEM, XL-30E Philips Co. Holland) and transmission electron microscope (FEI TECNAI G2 TEM) were used to analyze the morphology of the nanomaterials and the fiber distribution and diameter of the electrospun polymer nanocomposites. While the $\mathrm{X}$-ray diffractometer (Mini Flex 2, Rigaku equipped with Nickel filtered $\mathrm{CuK} \alpha$ radiation $(\lambda=0.1564 \mathrm{~nm})$ operated at $30 \mathrm{~V}$ and $15 \mathrm{~mA})$ scans the nanopowders and nanocomposites during $2 \theta, 10-30^{\circ}$ at a scanning speed of $1.8^{\circ} / \mathrm{min}$, FTIR spectroscope (PerkinElmer Spectrum 400 spectrophotometer) measures the absorbance during $400-4000 \mathrm{~cm}^{-1}$ wavelength at a resolution of $2 \mathrm{~cm}^{-1}$. Piezoelectric measurements were carried out per the established protocol [21-23] by which the electroded sample was kept above a shaker and under a constant load, and vibrations were induced on it using a frequency generator, amplifier and vibrating shaker in series. PENG was designed by silver electroding on both sides of the sample and attaching copper wires. The fibers were carefully embedded in specific polymer sheets without making the circuit closed. When the frequency generator sends vibrations to the sample, depending on the stress on it, the sample vibrates and produces piezoelectric output signals. Resistor box adjusts the resistance value to obtain maximum output performance and the power achieved can be calculated. Piezoelectric properties of the samples were correlated with the crystallinity data obtained from the differential scanning calorimetric analysis (DSC). DSC 8500 Perkin Elmer instrument measured the heating and cooling cycles of the fibers at a temperature range of 20 to $200{ }^{\circ} \mathrm{C}$ at $10{ }^{\circ} \mathrm{C} / \mathrm{min}$. The overall crystallinity values of the samples were directly proportional to the piezoelectric output voltage. While the thermal stability of $10 \mathrm{mg}$ of the fiber samples was tested using Perkin Elmer TGA4000 thermogravimetric analyzer, at a temperature range of $30^{\circ} \mathrm{C}$ to $800^{\circ} \mathrm{C} @$ $10{ }^{\circ} \mathrm{C}$ per minute (in nitrogen), the mechanical properties of the fibers (rectangular pieces of $2 \mathrm{~cm} \times 5 \mathrm{~cm}$ 
dimensions and $0.7 \mathrm{~mm}$ average thickness) were tested using Lloyd materials testing instrument by AMETEK. The sample was pulled at a constant rate of $2 \mathrm{~mm} / \mathrm{min}$, thus generating a strain Vs. stress graph that enables the derivation of the tensile strength, and Young's modulus. Energy storage properties of the electrospun fibers were tested by exploring the dielectric properties using broadband dielectric/impedance spectroscopeNovocontrol at a frequency range of $10^{1}$ to $10^{7}$.

\section{Results and discussion}

\subsection{Morphology and structural analysis of the nanomaterials}

Figure 1 shows the SEM and TEM morphologies of the nanomaterials $\mathrm{FeO}, \mathrm{ZnO}$ and $\mathrm{FeO} / \mathrm{ZnO}$ and their elemental analyses. Hydrothermal reaction generates $\mathrm{ZnO}$ as flowers according to the Ostwald ripening mechanism [27]. MEA forms an intermediate complex with the Zinc precursor and it forms rod like structures. The rod like structures further grow in to the form of flower like structures with typical sizes $\leq 1 \mu \mathrm{m}$. For the pure $\mathrm{ZnO}$, the average diameter of the flower shows $400 \mathrm{~nm}$ whereas for the $\mathrm{FeO}$, platelike sheets are observed with an average size of 220 $\mathrm{nm}$. For the $\mathrm{FeO} / \mathrm{ZnO}$ hybrid material, the average diameter of the nanoparticle became $280 \mathrm{~nm}$, with a slightly deformed flower like appearance. This is because during the hybrid particle synthesis, the $\mathrm{ZnO}$ flowers are formed on the $\mathrm{FeO}$ particles, since the hydrothermal reaction of the former was performed in the presence of the latter [28].

The EDX spectra shown in Fig. $1 \mathrm{~g}$ to $\mathrm{i}$ give information about the elements present in the synthesized filler particles. It is clear that the elements $\mathrm{Zn}$, and $\mathrm{Fe}$ are present in the $\mathrm{ZnO}$ and $\mathrm{FeO}$ samples, respectively, whereas both elements are observed in the hybrid. Absence of any other elements confirm the purity of the samples. In addition, the elemental composition of the samples are given in the insets that also confirms the structural integrity of the samples.

\subsection{Morphology and structural analysis of the electrospun nanocomposites}

Electrospun fibers of the polymer nanocomposites are investigated for their morphology and average fiber diameter. Figure 2 shows the SEM images of defect free fibers without the formation of beads, attributed to the optimum concentration of the PVDF solution used for electrospinning. Nanoparticles are embedded within the polymer chains and thus are not visible in the SEM images. The average fiber diameters are calculated from the diameter distribution curves, provided in the insets of the SEM images. The average diameter values observed are $249 \pm 109$ $\mathrm{nm}$ for the neat PVDF, $324 \pm 148 \mathrm{~nm}$ for the PVDF$\mathrm{FeO}, 442 \pm 268 \mathrm{~nm}$ for the PVDF-ZnO, $412 \pm 167 \mathrm{~nm}$ for PVDF-FeO/ZnO at $1 \mathrm{wt} \%$ and $417 \pm 188 \mathrm{~nm}$ for the PVDF-FeO/ZnO at $3 \mathrm{wt} \%$ nanocomposites. It's clear that the average fiber diameter enhances for the nanocomposites due to enhanced viscosity and networking effect attributed to the good distribution of filler particles [29]. For the hybrid composites, much variation in fiber diameters are not observed due to the similar levels of filler dispersion within the PVDF polymer.

Structural information of the PVDF composite fibers are explored from the FTIR and XRD studies as evidenced from the Fig. 3. The FTIR spectra of the PVDF nanocomposites in Fig. 3a shows the absorbance peak at $842 \mathrm{~cm}^{-1}$, which is due to the presence of crystalline $\beta$-phase [30]. Absence of peaks at 976 and $766 \mathrm{~cm}^{-1}$ corresponds to the absence of $\alpha$-phase. While the peak at $1176 \mathrm{~cm}^{-1}$ corresponds to the $\beta$ phase, the peak at $1398 \mathrm{~cm}^{-1}$ is due to the $\gamma$-phase $[31,32]$. The crystalline $\beta$-phase proportions for the PVDF fibers can be calculated from the absorbance values at $860 \mathrm{~cm}^{-1}\left(A_{\beta}\right)$ and $760 \mathrm{~cm}^{-1}\left(A_{\alpha}\right)$ as per the following equation [21].

$F(\beta)=\frac{A_{\beta}}{1.26 A_{\alpha}+A_{\beta}}$

The value enhances from 21.36 for the PVDF to 22.39 for the PVDF composites containing hybrid filler materials. This shows the increase in $\beta$-phase with the introduction of metal oxide nanomaterial (crystallinity value given in Table 3).

Crystallization behavior of the PVDF composites can also be explored from these spectral analyses. XRD pattern for the fibers given in Fig. $3 b$ demonstrates the semicrystalline nature of the PVDF with peak positions at $18.6^{\circ}$ and $20.4^{\circ}$ respectively attributed to the (020) and (021)/(201) crystal planes of $\alpha$ - 

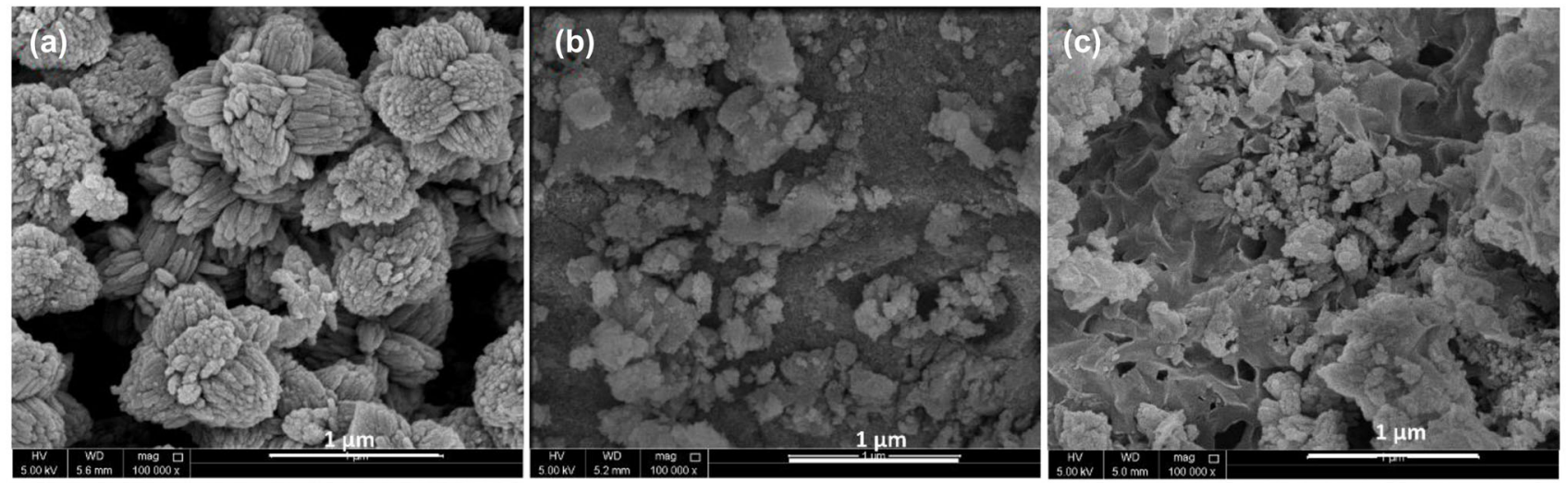

(d)
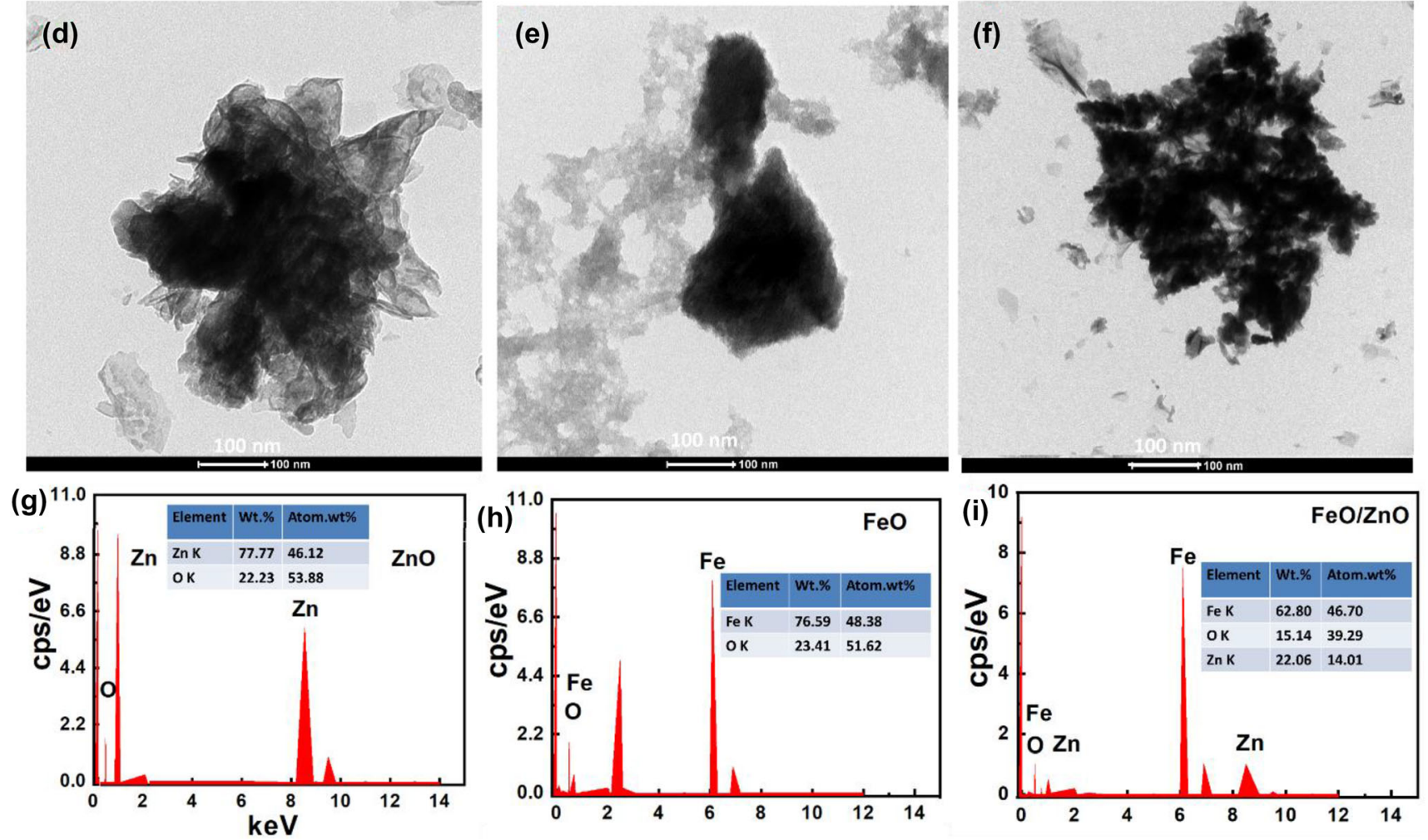

Fig. 1 SEM images of $\mathbf{a} \mathrm{ZnO}, \mathbf{b} \mathrm{FeO}$ and $\mathbf{c} \mathrm{FeO} / \mathrm{ZnO}$; TEM images of $\mathbf{d} \mathrm{ZnO}$, e $\mathrm{FeO}$ and $\mathbf{f} \mathrm{FeO} / \mathrm{ZnO}$ and $\mathrm{EDX}$ spectra of $\mathbf{g} \mathrm{ZnO}$, $\mathbf{h} \mathrm{FeO}$ and $\mathbf{i} \mathrm{FeO} / \mathrm{ZnO}$ nanomaterials

phase of the PVDF [33]. The peak at $20.4^{\circ}$ also corresponds to the (110) and (200) crystal planes of the $\beta$ phase PVDF. A prominent peak at $36.4^{\circ}$ also appears with the introduction of nanoparticles, which is due to the (020) and (101) crystal planes of the $\beta$-phase. Peaks at $40.8^{\circ}$ and $56.8^{\circ}$ can be the secondary peaks from overtone bands. Crystalline fractions within the composite fibers can be analyzed from the variation in peak intensities with the introduction of filler particles. It's clear from the figure that the electroactive phase enhances considerably with the introduction of metal oxides, and $\beta$-phase transformation also exist [34, 35]. This is because of the influence of semiconducting fillers in determining the diploe alignments within the PVDF chains [20].

\subsection{Thermal and mechanical stability of the electrospun nanocomposites}

Thermogravimmetric and derivative thermogravimmetric curves obtained for the PVDF nanocomposite fibers are provided in Fig. 4. It is observed that the PVDF composites are stable up to $430{ }^{\circ} \mathrm{C}$ and starts degradation after this point. The decomposition 

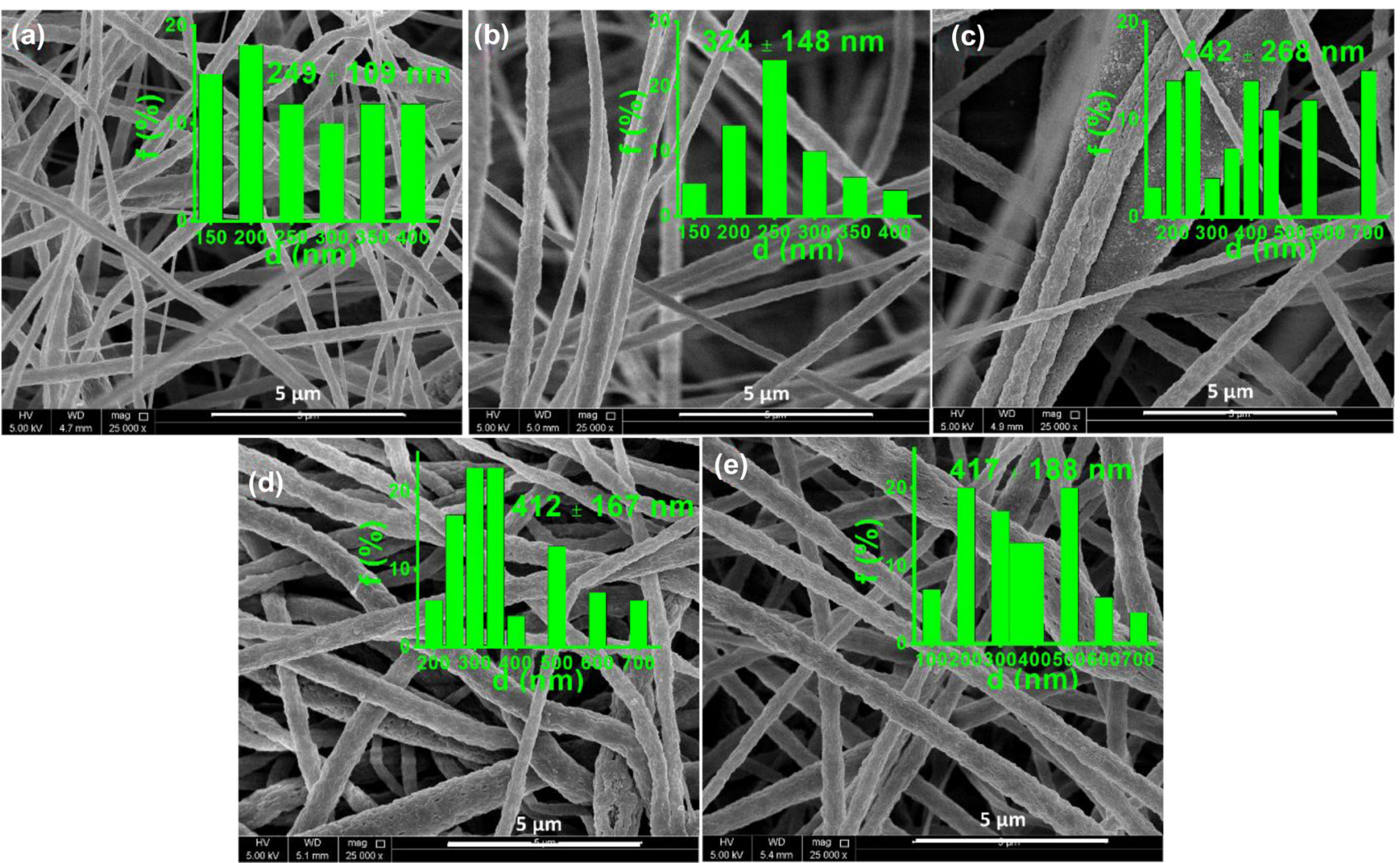

Fig. 2 SEM images of a neat PVDF, b PVDF-FeO, c PVDF-ZnO, d PVDF-FeO/ZnO (1 wt\%) and e PVDF-FeO/ZnO (3 wt\%) electrospun fibers; inset shows the average fiber diameter distribution plots

temperature for the neat PVDF is at $462{ }^{\circ} \mathrm{C}$, whereas this value enhances with the introduction of $\mathrm{ZnO}$ and $\mathrm{FeO}$ filler particles [36]. With the addition of $\mathrm{FeO}$ and $\mathrm{ZnO}$, the decomposition temperature becomes respectively $470.58{ }^{\circ} \mathrm{C}$ and $469.92{ }^{\circ} \mathrm{C}$, indicating the similar effect of both nanofillers. This is because of the high distribution of the filler materials within the polymer medium and the restricted movement of PVDF chains by the nanomaterials. The well distributed nanoparticles cause for the higher crosslink density, stronger adhesion with the polymer chains and improved crystallinity, leading to the high thermal stability [37]. However the hybrid composite enhanced thermal stability to much higher value reaching up to $482.6{ }^{\circ} \mathrm{C}$ for the composite containing $\mathrm{FeO} / \mathrm{ZnO}$ at $1 \mathrm{wt} \%$.

Table 1 shows the tensile behavior of the fiber samples in terms of tensile strength, Young's modulus and stiffness values. All values show a regular increase with the addition of nanoparticles and also with increased concentration. When the hybrid filler is compared with the $\mathrm{FeO}$ and $\mathrm{ZnO}$ individual composites, higher tensile values are achieved due to the filler synergy. Both semiconducting metal oxide nanoparticles are oriented in all directions within the PVDF polymeric chain, and forms interconnected networks [38]. The stress transfers from the polymer to filler networks during the tensile measurements. High stiffness is also observed for the PVDF composite containing $3 \mathrm{wt} \% \mathrm{FeO} / \mathrm{ZnO}$ combination.

\subsection{Piezoelectric properties and correlation with crystallinity}

Piezoelectric properties of the composite fibers are tested by preparing the nanogenerators with the help of aluminium foil, copper wires and PET substrates [11]. Rectangular fibers of $2.5 \mathrm{~cm} \times 2.5 \mathrm{~cm}$ dimension were sandwiched between the aluminium foils and copper wires were connected using conducting tape on both sides. Double side adhesive tapes were used to wrap PET substrates on both sides of the sample. This PENG is tested for the piezoelectric output voltage as per the established protocol [21], explained in the Sect. 2. Figure 5 represents the piezoelectric 

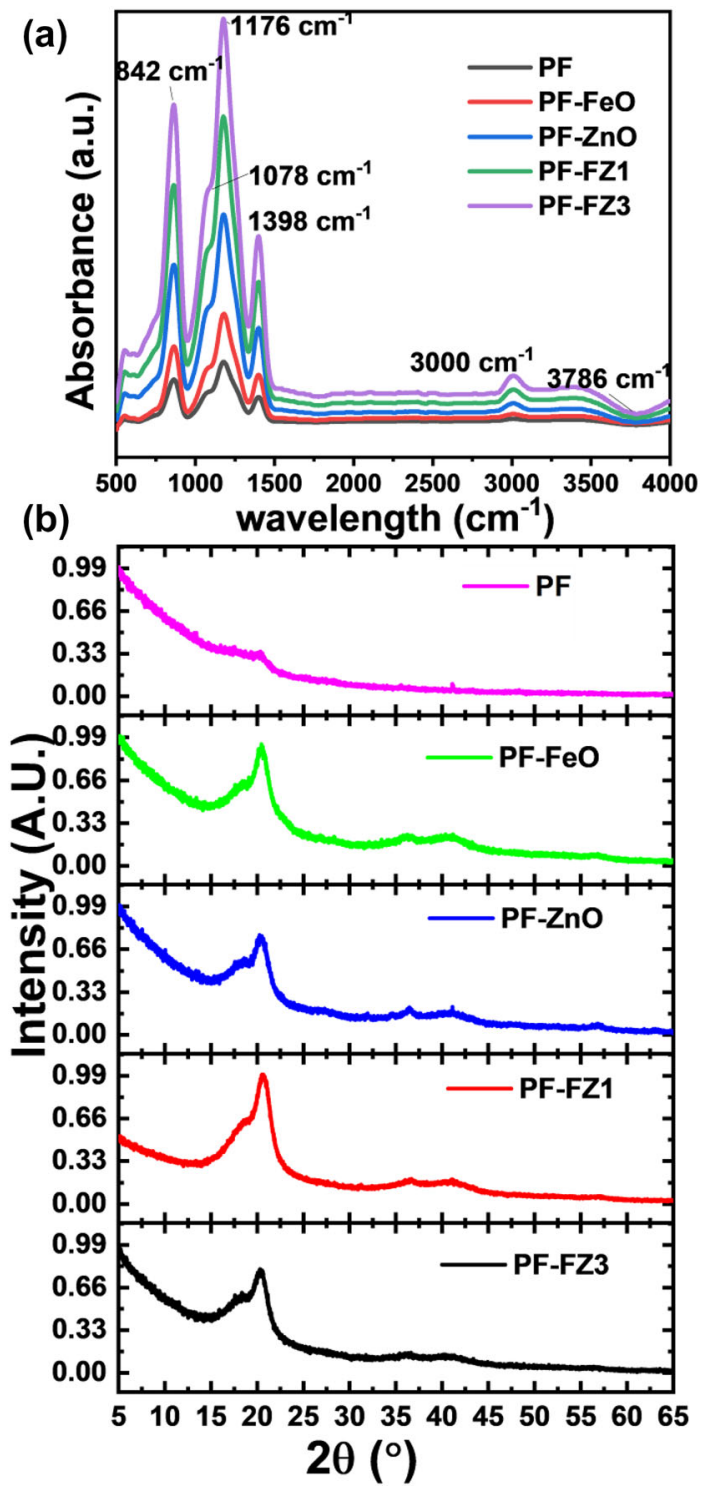

Fig. 3 a FTIR and $\mathbf{b}$ XRD spectra of PVDF nanocomposite fibers

output voltage obtained for all the PVDF fibers when placed under a load of $2.5 \mathrm{~N}$ and at $1 \mathrm{M} \Omega$ resistance. With increased vibrational frequency, the output voltage increases and reaches a point at which the voltage levels off.

The PENG made from the $3 \mathrm{wt} \%$ of the hybrid nanofiller shows the highest output compared to all the other composite fibers, attributed to the reinforcing effect achieved by the filler synergy. The metal oxides form interconnected networks throughout the sample and allows the mechanical vibration transport and the electrical energy transfer [39]. This is happening as the $\mathrm{FeO}$ and $\mathrm{ZnO}$ filler materials have unique dipole alignment and ferroelectric characteristics helping in generating the piezoelectricity [21, 22]. Electrospinning allows the dipole alignment, which is responsible for the piezoelectricity [11]. The dipoles in the $\mathrm{ZnO}$ and $\mathrm{FeO}$ also contribute to the enhanced piezoelectric response. Moreover, the nanoparticles act as stress connecting points, thus separating the electrospun PVDF fibers to many segments. This causes increased local deformations and thus again results in the high piezoelectric output voltage.

Figure 6a compares the maximum output voltages obtained for all the samples, and shows the flexibility of the PENG. The voltage variation of the PVDF/ $\mathrm{FeO} / \mathrm{ZnO}$ at $3 \mathrm{wt} \%$ composite fiber for 2000 cycles is also represented in the Fig. $6 \mathrm{~b}$. In all cases, the hybrid composite is identified to have good response, which is attributed to the following factors. When the $\mathrm{ZnO}$ and $\mathrm{FeO}$ hybrid particles are present in the composites, the $\beta$-phase crystallinity was more as evident from the FTIR and XRD results. This is further explained with the help of DSC studies and it is believed that the hybrid fillers cause $\beta$-phase nucleation [40]. It is also established that the interfacial effects and electroactive phase nucleation happen in the presence of ferrite nanoparticles with magnetic characteristics such as $\mathrm{FeO}$ [41]. The FeO has surface charges and its crystalline nature contribute to good piezoelectric property enhancement. Higher mechanical stability is achieved for the hybrid filler reinforced samples, due to the networking effect and this enhances the stress of fibers and the PVDF local stress. Moreover, the semiconducting nanoparticles can form connections or conducting paths throughout for the induced charge transfer within the polymer nanocomposites. The significance of current research in comparison with the published studies is illustrated in the following table (Table 2). It is clear that the advantage of the current research lays in the less concentration of nanomaterial used, absence of complicated nanomaterial processing and in the high voltage obtained.

Figure 7 illustrates the heating and cooling curves of the PVDF composite fibers, investigated by the DSC analysis. The melting and crystallization temperatures of the composites and the crystallinity are illustrated in the Table 3. The melting temperature of neat PVDF increases from 158.92 to $161.07{ }^{\circ} \mathrm{C}$, when 

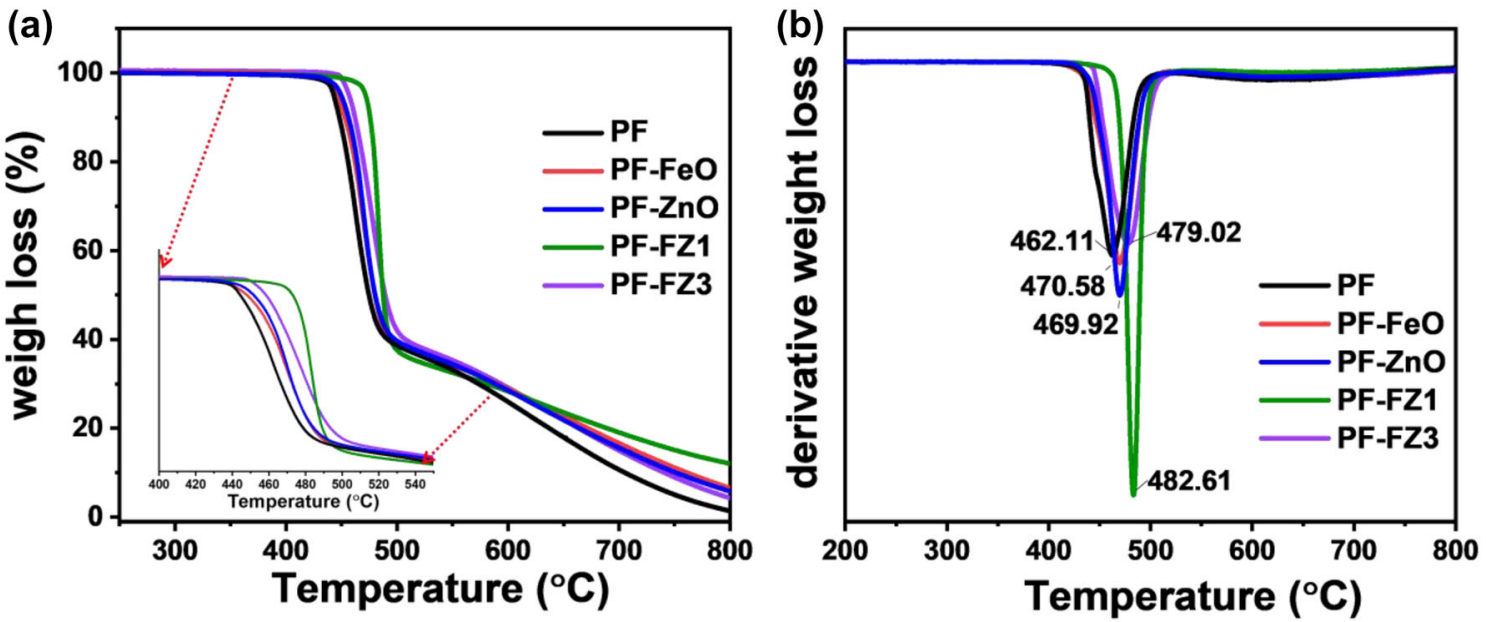

Fig. 4 a TGA and $\mathbf{b}$ DTA curves of PVDF nanocomposite fibers

Table 1 Tensile properties of the PVDF nanocomposite fibers

\begin{tabular}{lccc}
\hline Samples & Young's modulus $(\mathrm{MPa})$ & Tensile strength & Stiffness $(\mathrm{N} / \mathrm{mm})$ \\
\hline PF & $6.95 \pm 1.5$ & $0.156 \pm 0.12$ & $0.52 \pm 0.05$ \\
PF-FeO & $8.87 \pm 0.52$ & $0.192 \pm 0.15$ & $0.63 \pm 0.10$ \\
PF-ZnO & $9.71 \pm 3.5$ & $0.313 \pm 0.09$ & $0.69 \pm 0.08$ \\
PF-FZ1 & $12.54 \pm 2.1$ & $0.370 \pm 0.10$ & $0.81 \pm 0.08$ \\
PF-FZ3 & $17.52 \pm 2.5$ & $0.475 \pm 0.11$ & $1.03 \pm 0.12$ \\
\hline
\end{tabular}

(a)

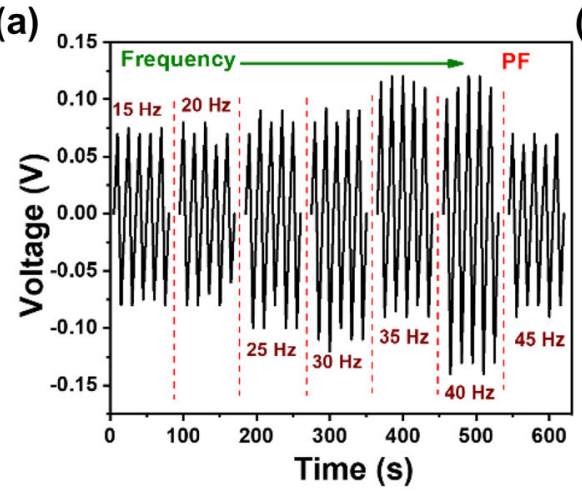

(b)

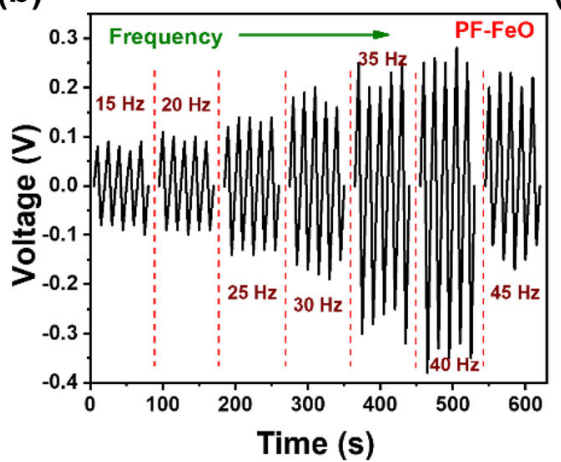

(c)

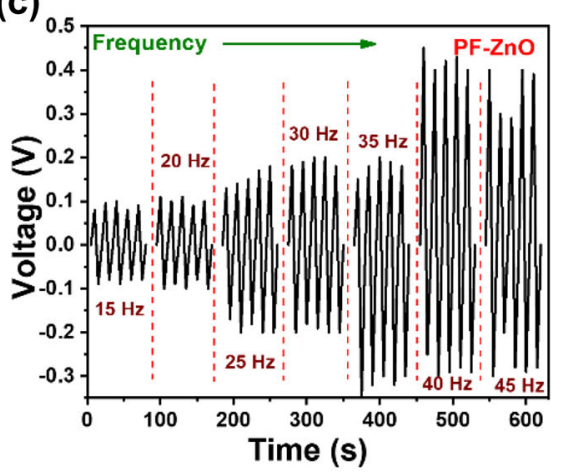

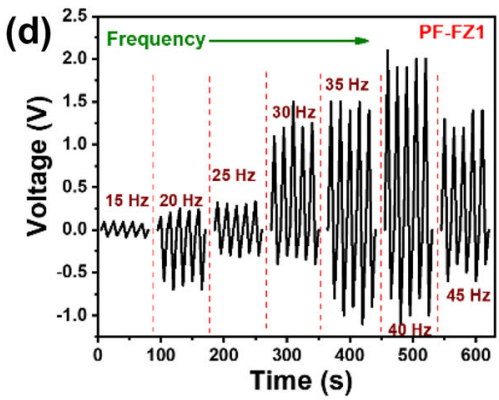

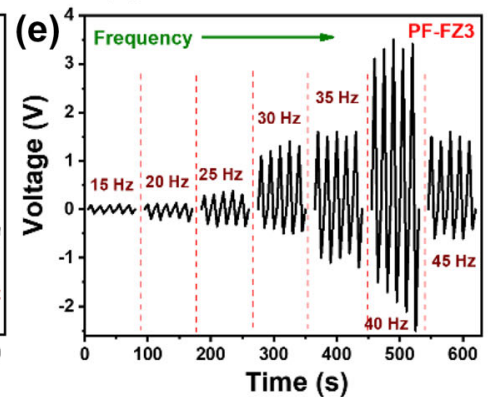

Fig. 5 Piezoelectric output voltage variation with frequency a PVDF, b PVDF-FeO, c PVDF-ZnO, d PVDF-FeO/ZnO at 1 wt $\%$ and e PVDF-FeO/ZnO at $3 \mathrm{wt} \%$ composite fibers 

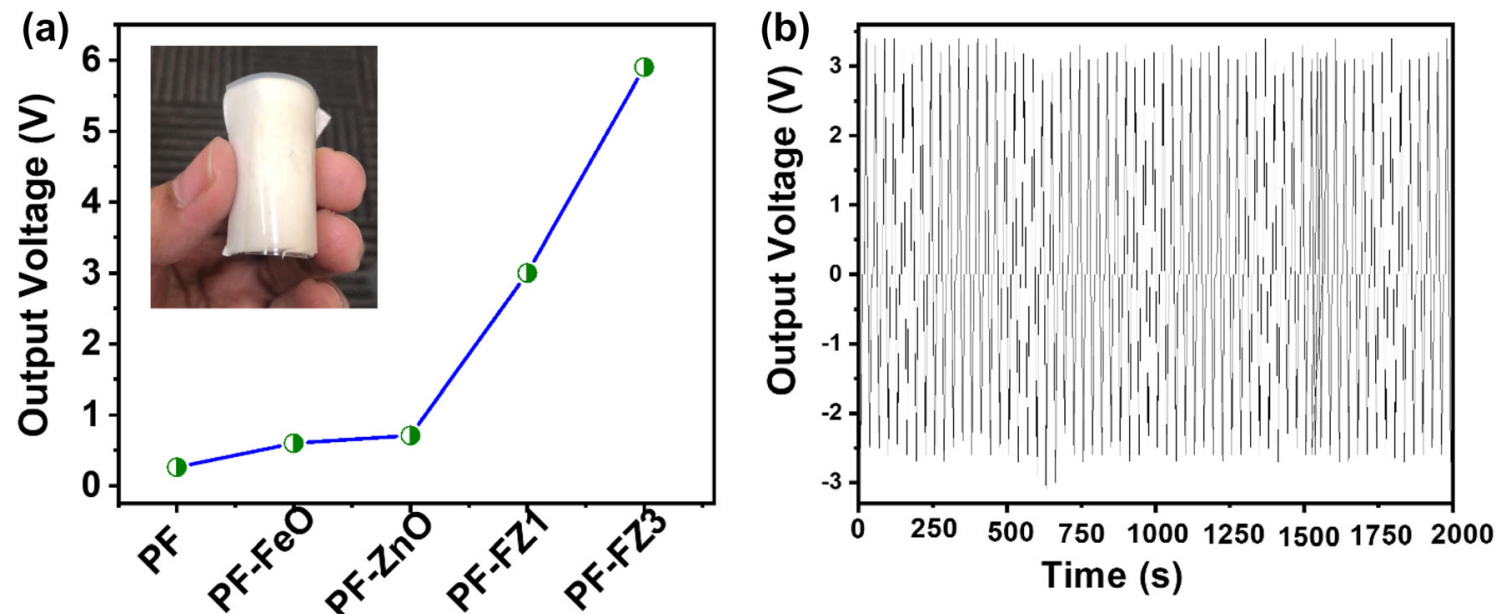

Fig. 6 a Peak to peak output voltage for all samples (inset shows the flexibility), b durability of the output voltage with time

Table 2 Comparison of the current piezoelectric voltage achieved with the published values

\begin{tabular}{lllll}
\hline Nanocomposite & Fabrication & Concentration & Output voltage & References \\
\hline $\mathrm{PVDF} /$ graphene/BaTiO & & Electrospinning & $0.15 \mathrm{wt} \%$ (graphene) $/ 15 \mathrm{wt} \% \mathrm{BaTiO}_{3}$ & $11 \mathrm{~V}$ \\
$\mathrm{PVDF} / \mathrm{Fe}-\mathrm{ZnO}$ & Gamma irradiation of casted films & $2 \mathrm{wt} \%$ & $2.4 \mathrm{~V}$ & {$[11]$} \\
$\mathrm{PVDF} / \mathrm{Cloisite} 30 \mathrm{~B}$ & Electrospinning & $15 \mathrm{wt} \%$ & $70 \mathrm{~V}$ & {$[23]$} \\
$\mathrm{PVDF} / \mathrm{TiO}_{2}-\mathrm{Fe}_{3} \mathrm{O}_{4}-$ & Electrospinning & $2 \mathrm{wt} \%$ & $51.42 \mathrm{mV} / \mathrm{N}$ & {$[39]$} \\
$\mathrm{MWCNT}$ & & & $5.9 \mathrm{~V}$ \\
Current study & Electrospinning & $3 \mathrm{wt} \%$ & \\
\hline
\end{tabular}

Fig. 7 a Heating and b Cooling curves of PVDF nanocomposite fibers (a)

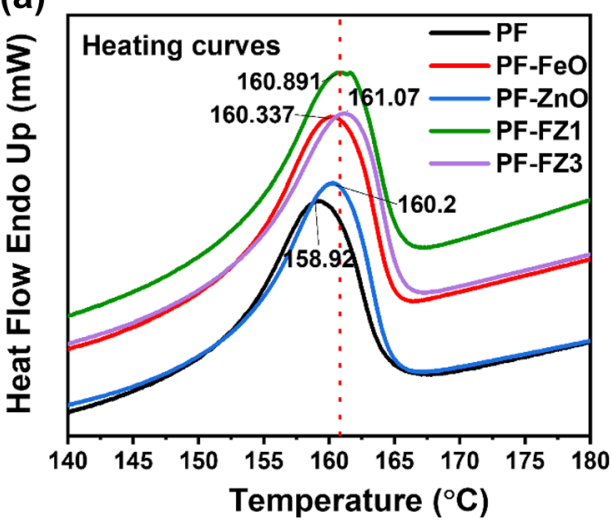

(b)

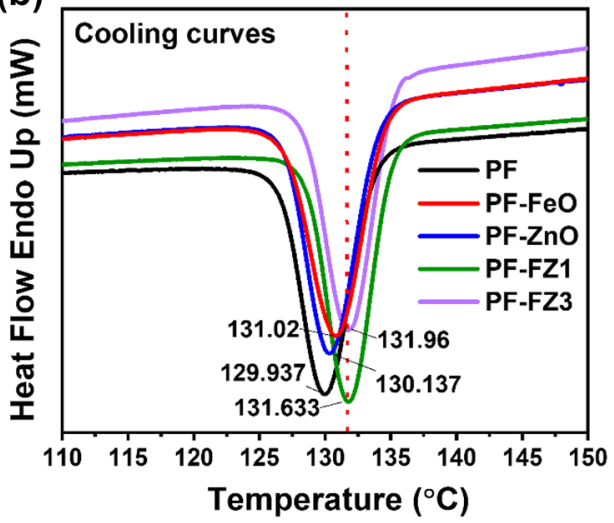

the $\mathrm{FeO} / \mathrm{ZnO}$ are added to the polymer. This is attributed to the increased interaction between the polymer and the metal oxide nanomaterials, coming from the good dispersion of metal oxides and its reinforcing effect. Crystallization temperature of the PVDF nanocomposites also follow similar trend of variation indicating the change in crystallization behavior by the introduction of metal oxides. The typical reasons for the improved crystallization behavior of the nanocomposites are the high viscosity and the increased entanglement [42].

Table 3 shows the melting and crystallization temperatures of the PVDF composites and the crystallinity values of the samples. Crystallinity $\left(X_{c}\right)$ of the fibers are calculated from the melting enthalpy 
Table 3 Crystallization and melting parameters of PVDF nanocomposite fibers

\begin{tabular}{lllll}
\hline Samples & $T_{\mathrm{m}}\left({ }^{\circ} \mathrm{C}\right)$ & $T_{\mathrm{c}}\left({ }^{\circ} \mathrm{C}\right)$ & Crystallinity from DSC $\mathrm{X}_{\mathrm{c}}(\%)$ & Crystallinity from FTIR \\
\hline $\mathrm{PF}$ & 158.92 & 129.94 & 33.31 & 21.36 \\
$\mathrm{PF}-\mathrm{FeO}$ & 160.34 & 131.02 & 33.96 & 21.95 \\
PF-ZnO & 160.20 & 130.14 & 33.15 & 21.54 \\
PF-FZ1 & 160.89 & 131.64 & 34.43 & 22.39 \\
PF-FZ3 & 161.07 & 131.96 & 34.03 & 22.17 \\
\hline
\end{tabular}

Fig. 8 a Dielectric constant and $\mathbf{b} \tan \delta$ values against frequency for the PVDF composite fibers
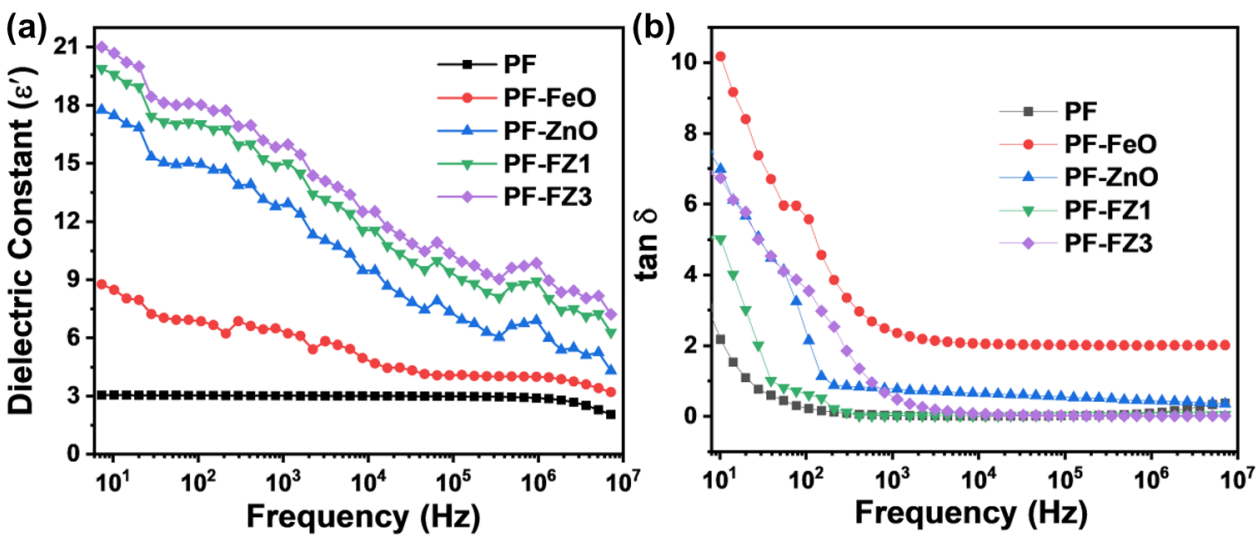

$\left(\Delta H_{\mathrm{m}}\right)$ value and weight fraction of filler particles $(\phi)$ in PVDF composites according to the following equation [43].

$X_{\mathrm{c}}=\left(\frac{\Delta H_{\mathrm{m}}}{\phi \Delta H_{\mathrm{m}}^{\circ}}\right) \times 100$

$\Delta H_{\mathrm{m}}^{\circ}$ is the melting enthalpy value for a typical $\alpha$ PVDF crystal and is fixed as $104.6 \mathrm{~J} / \mathrm{g}$.

The crystallinity values of the PVDF composite fibers corresponds to the crystalline regions of both $\alpha$ and $\beta$ phase [44]. From the values given in Table 3, a small but gradual increase of PVDF crystallinity with increase of filler concentration is observed, which can be due to the filler network formed within the polymer chains as a result of good dispersion. There is a small decrease in crystallinity when the higher concentration of $\mathrm{FeO} / \mathrm{ZnO}$ is considered, because of the hindered PVDF chain diffusion by the highly concentrated nanomaterials, preventing the crystallization processes [44].

\subsection{Dielectric properties of the nanocomposites}

The frequency dependent dielectric properties of the nanocomposites are demonstrated in the Fig. 8. The dielectric constant for the PVDF-FeO/ZnO sample at $3 \mathrm{wt} \%$ nanoparticle concentration shows a value of
21 , which is around 7 times higher than that of the neat polymer fibers. This enhancement in dielectric constant value for the nanocomposite is due to the Maxwell-Wagner-Sillars (MWS) effect by which the interfacial polarization occurs with the charge accumulation and short-range dipole dipole interactions at the PVDf/nanoparticle interface with the electrical field. With frequency, the number of aligned dipoles decreases and thus the dielectric constant. At higher frequencies, the dipoles lag with the applied electric filed as well. In addition to the MWS effect, the total interfacial area per unit volume of the nanoparticle also influences the dielectric constant value enhancement. Higher interfacial area reduces the interparticle distance in unit volume, leading to inter particle coupling and enhanced average polarization [5]. Moreover, the beta phase of the PVDF is improved by the addition of $\mathrm{FeO}$ and $\mathrm{ZnO}$ nanomaterials, which also contribute towards higher dielectric constant. Figure $8 \mathrm{~b}$ shows the variation in loss tangent (increasing trend with the nanoparticle loading) with frequency. In this figure, a relaxation region of $10^{2}$ to $10^{5} \mathrm{~Hz}$ frequency is observed which can be attributed to the $\alpha-C$ type of relaxation [40]. 


\section{Conclusions}

This article reports a simple and convenient method of electrospinning to develop flexible PENGs from PVDF and metal oxide hybrid nanomaterials $(\mathrm{FeO} /$ $\mathrm{ZnO}$ ) with good piezoelectric and dielectric properties. The composite fibers show defect free morphology with metal oxides grafted physically within. Increased fiber diameter and defect free morphology confirm the good dispersibility of the nanomaterials, and thus crystallinity enhancement is anticipated. While the mechanical strength improves three times for the PVDF-FeO/ZnO (3 wt\%) compared to the neat polymer, the thermal degradation temperature shows a difference of approximately $20{ }^{\circ} \mathrm{C}$. This shows the mechanical and thermal stability of the fibers. Also, the dielectric permittivity and piezoelectric output voltage of the hybrid filler composite respectively increase from 3 to 21 and 0.2 to $5.9 \mathrm{~V}$, with the filler introduction. A good correlation between the electroactive phase formation and piezoelectric output voltage generation is observed for the PVDF composite fibers.

\section{Acknowledgements}

We would like to thank the Qatar University' Central Laboratories Unit (QUCLU) for their assistance with the SEM and TEM measurements of the samples.

\section{Funding}

Open access funding provided by the Qatar National Library. This publication was made possible by UREP Grant 24-142-1-032 from the Qatar National Research Fund (a member of Qatar Foundation). The statements made herein are solely the responsibility of the authors.

\section{Declaration}

Conflict of interest The authors declare that they have no conflict of interest.

Consent to participate All authors have the consent to participate.

Consent for publication All authors have the consent to publish.
Open Access This article is licensed under a Creative Commons Attribution 4.0 International License, which permits use, sharing, adaptation, distribution and reproduction in any medium or format, as long as you give appropriate credit to the original author(s) and the source, provide a link to the Creative Commons licence, and indicate if changes were made. The images or other third party material in this article are included in the article's Creative Commons licence, unless indicated otherwise in a credit line to the material. If material is not included in the article's Creative Commons licence and your intended use is not permitted by statutory regulation or exceeds the permitted use, you will need to obtain permission directly from the copyright holder. To view a copy of this licence, visit http://creativecommons.org/licen ses/by $/ 4.0 /$.

\section{References}

1. Y. Liang, C.Z. Zhao, H. Yuan, Y. Chen, W. Zhang, J.Q. Huang, D. Yu, Y. Liu, M.M. Titirici, Y.L. Chueh, H. Yu, A review of rechargeable batteries for portable electronic devices. InfoMat 1(1), 6-32 (2019 Mar)

2. K. Yu, X. Pan, G. Zhang, X. Liao, X. Zhou, M. Yan, L. Xu, L. Mai, Nanowires in energy storage devices: structures, synthesis, and applications. Adv. Energy Mater. 8(32), 1802369 (2018)

3. Y. Li, J. Yang, J. Song, Design structure model and renewable energy technology for rechargeable battery towards greener and more sustainable electric vehicle. Renew. Sustain. Energy Rev. 74, 19-25 (2017)

4. J. Briscoe, S. Dunn, Piezoelectric nanogenerators - a review of nanostructured piezoelectric energy harvesters. Nano Energy 1, 15-29 (2015)

5. B. Dutta, E. Kar, N. Bose, S. Mukherjee, NiO@ SiO2/PVDF: A flexible polymer nanocomposite for a high performance human body motion-based energy harvester and tactile e-skin mechanosensor. ACS Sustain. Chem. Eng. 6(8), 10505-16 (2018)

6. Q. Zheng, H. Zhang, H. Mi, Z. Cai, Z. Ma, S. Gong, Highperformance flexible piezoelectric nanogenerators consisting of porous cellulose nanofibril (CNF)/poly (dimethylsiloxane)(PDMS) aerogel films. Nano Energy. 26, 504-12 (2016)

7. D. Ponnamma, H. Parangusan, A. Tanvir, M.A. AlMa'adeed, Smart and robust electrospun fabrics of piezoelectric polymer nanocomposite for self-powering electronic textiles. Mater. Des. 15, 108176 (2019) 
8. K.T. Arul, M.R. Rao, Ferroelectric properties of flexible PZT composite films. J. Phys. Chem. Solids 1, 146:109371 (2020 Nov)

9. K.T. Arul, M. Ramanjaneyulu, M.R. Rao, Energy harvesting of PZT/PMMA composite flexible films. Curr. Appl. Phys. 19(4), 375-80 (2019)

10. D. Ponnamma, M.A. Al-Maadeed, Influence of BaTiO 3/white graphene filler synergy on the energy harvesting performance of a piezoelectric polymer nanocomposite. Sustain. Energy Fuels 3(3), 774-785 (2019)

11. K. Shi, B. Sun, X. Huang, P. Jiang, Synergistic effect of graphene nanosheet and $\mathrm{BaTiO} 3$ nanoparticles on performance enhancement of electrospun PVDF nanofiber mat for flexible piezoelectric nanogenerators. Nano Energy. 52, 153-62 (2018)

12. G. Wang, J. Liu, X. Liu, W. Feng, J. Yang, Extensional vibration characteristics and screening of polarization charges in a $\mathrm{ZnO}$ piezoelectric semiconductor nanofiber. J. Appl. Phys. 124(9), 094502 (2018)

13. I.H. Lone, J. Aslam, N.R. Radwan, A.H. Bashal, A.F. Ajlouni, A. Akhter, Multiferroic, ABO 3 transition metal oxides: a rare interaction of ferroelectricity and magnetism. Nanoscale Res. Lett. 14(1), 1-2 (2019)

14. L. Lu, W. Ding, J. Liu, B. Yang, Flexible PVDF based piezoelectric nanogenerators. Nano Energy 6, 105251 (2020 Aug)

15. D. Ponnamma, A. Erturk, H. Parangusan, K. Deshmukh, M.B. Ahamed, M.A. Al-Maadeed, Stretchable quaternary phasic PVDF-HFP nanocomposite films containing graphenetitania-SrTiO3 for mechanical energy harvesting. Emerg. Mater. 1(1-2), 55-65 (2018)

16. N. Soin, D. Boyer, K. Prashanthi, S. Sharma, A.A. Narasimulu, J. Luo, T.H. Shah, E. Siores, T. Thundat, Exclusive self-aligned $\beta$-phase PVDF films with abnormal piezoelectric coefficient prepared via phase inversion. Chem. Commun. 51(39), 8257-8260 (2015)

17. M.A. Sibeko, M.L. Saladino, F. Armetta, A. Spinella, A.S. Luyt, Effect of preparation method on the properties of poly (methyl methacrylate)/mesoporous silica composites. Emerg. Mater. 2(3), 363-70 (2019)

18. A.A. Issa, M.A. Al-Maadeed, A.S. Luyt, D. Ponnamma, M.K. Hassan, Physico-mechanical, dielectric, and piezoelectric properties of PVDF electrospun mats containing silver nanoparticles. C-J. Carbon Res. 3(4), 30 (2017)

19. K.M. Nam, W.S. Seo, H. Song, J.T. Park, Non-native transition metal monoxide nanostructures: unique physicochemical properties and phase transformations of $\mathrm{CoO}, \mathrm{MnO}$ and ZnO. NPG Asia Mater. 9(3), e364 (2017)

20. D. Ponnamma, O. Aljarod, H. Parangusan, M.A. Al-Maadeed, Electrospun nanofibers of PVDF-HFP composites containing magnetic nickel ferrite for energy harvesting application. Mater. Chem. Phys. 239, 122257 (2020)

21. H. Parangusan, D. Ponnamma, M.A. Al-Maadeed, Stretchable electrospun PVDF-HFP/Co-ZnO nanofibers as piezoelectric nanogenerators. Sci. Rep. 8(1), 1-1 (2018)

22. H. Parangusan, D. Ponnamma, M.A. AlMaadeed, Flexible trilayer piezoelectric nanogenerator based on PVDF-HFP/Nidoped $\mathrm{ZnO}$ nanocomposites. RSC Adv. 7(79), 50156-50165 (2017)

23. H. Parangusan, D. Ponnamma, M.A. AlMaadeed, Investigation on the effect of $\gamma$-irradiation on the dielectric and piezoelectric properties of stretchable $\mathrm{PVDF} / \mathrm{Fe}-\mathrm{ZnO}$ nanocomposites for self-powering devices. Soft Matter 14(43), 8803-8813 (2018)

24. H. Wang, X. Liang, J. Wang, S. Jiao, D. Xue, Multifunctional inorganic nanomaterials for energy applications. Nanoscale 12(1), 14-42 (2020)

25. H.N. Ji, Y.P. Ok, W.P. Tai, J.H. Seol, J.S. Lee, Preparation of lead-free $(\mathrm{K}, \mathrm{Na}) \mathrm{Nb3}$-LiSbO3 ceramics with high piezoelectric constants by $\mathrm{FeO}$ doping. J. Kor. Phys. Soc. 56(4), 1156-1159 (2010)

26. P. Rong, S. Ren, Q. Yu, Fabrications and applications of $\mathrm{ZnO}$ nanomaterials in flexible functional devices-a review. Crit. Rev. Anal. Chem. 49(4), 336-49 (2019)

27. H. Parangusan, D. Ponnamma, M.A. Al-Maadeed, A. Marimuthu, Nanoflower-like yttrium-doped $\mathrm{ZnO}$ photocatalyst for the degradation of methylene blue dye. Photochem. Photobiol. 94(2), 237-246 (2018)

28. A. Barhoum, J. Melcher, G. Van Assche, H. Rahier, M. Bechelany, M. Fleisch, D. Bahnemann, Synthesis, growth mechanism, and photocatalytic activity of Zinc oxide nanostructures: porous microparticles versus nonporous nanoparticles. J. Mater. Sci. 52(5), 2746-62 (2017)

29. R. Augustine, P. Dan, A. Sosnik, N. Kalarikkal, N. Tran, B. Vincent, S. Thomas, P. Menu, D. Rouxel, Electrospun poly (vinylidene fluoride-trifluoroethylene)/zinc oxide nanocomposite tissue engineering scaffolds with enhanced cell adhesion and blood vessel formation. Nano Research 10(10), 3358-76 (2017)

30. A. Al-Saygh, D. Ponnamma, M.A. AlMaadeed, P.P. Vijayan, A. Karim, M.K. Hassan, Flexible pressure sensor based on PVDF nanocomposites containing reduced graphene oxidetitania hybrid nanolayers. Polymers 9(2), 33 (2017 Feb)

31. X. Cai, T. Lei, D. Sun, L. Lin, A critical analysis of the $\alpha, \beta$ and $\gamma$ phases in poly (vinylidene fluoride) using FTIR. RSC Adv. 7(25), 15382-15389 (2017)

32. E. Kar, N. Bose, S. Das, N. Mukherjee, S. Mukherjee, Enhancement of electroactive $\beta$ phase crystallization and dielectric constant of PVDF by incorporating $\mathrm{GeO} 2$ and $\mathrm{SiO}$ 
2 nanoparticles. Phys. Chem. Chem. Phys. 17(35), 22784-22798 (2015)

33. S. Tiwari, A. Gaur, C. Kumar, P. Maiti, Enhanced piezoelectric response in nanoclay induced electrospun PVDF nanofibers for energy harvesting. Energy 15, 171:485-492 (2019 Mar)

34. S. Dash, H.S. Mohanty, A. Kumar, R. Thomas, D.K. Pradhan, Ferroelectric ceramic dispersion to enhance the $\beta$ phase of polymer for improving dielectric and ferroelectric properties of the composites. Polym. Bull. 30, 1-20 (2020 Sep)

35. Y. Su, Y. Gu, H. Li, F. Geng, Ag-NBCTO-PVDF composites with enhanced dielectric properties. Mater. Lett. 185, 208-10 (2016)

36. C. Du, M. Li, M. Cao, S. Feng, H. Guo, B. Li, Enhanced thermal and mechanical properties of polyvinlydene fluoride composites with magnetic oriented carbon nanotube. Carbon. 126, 197-207 (2018)

37. P.C. Irwin, Y. Cao, A. Bansal, L.S. Schadler, Thermal and mechanical properties of polyimide nanocomposites. In: 2003 Annual report conference on electrical insulation and dielectric phenomena 2003 (pp. 120-123). IEEE

38. D. Ponnamma, O. Aljarod, H. Parangusan, M.A. Al-Maadeed, Reduction in piezoelectric voltage generation for the cerium doped nickel ferrite nanoparticles filled PVDF-HFP nanocomposites. Results Phys. 13, 102130 (2019)

39. A. Samadi, R. Ahmadi, S.M. Hosseini, Influence of TiO2Fe3O4-MWCNT hybrid nanotubes on piezoelectric and electromagnetic wave absorption properties of electrospun PVDF nanocomposites. Org. Electron. 1, 75:105405 (2019 Dec)
40. N. Maity, A. Mandal, A.K. Nandi, Hierarchical nanostructured polyaniline functionalized graphene/poly (vinylidene fluoride) composites for improved dielectric performances. Polymer 103, 83-97 (2016)

41. Z.W. Ouyang, E.C. Chen, T.M. Wu, Enhanced piezoelectric and mechanical properties of electroactive polyvinylidene fluoride/iron oxide composites. Mater. Chem. Phys. 149, 172-8 (2015)

42. W.Z. Lang, Y.J. Guo, L.F. Chu, Evolution of the precipitation kinetics, morphologies, permeation performances, and crystallization behaviors of polyvinylidenefluoride (PVDF) hollow fiber membrane by adding different molecular weight polyvinylpyrrolidone (PVP). Polym. Adv. Technol. 22(12), 1720-1730 (2011)

43. W. Ma, S. Chen, J. Zhang, X. Wang, Crystallization behavior and hydrophilicity of poly (vinylidene fluoride)(PVDF)/poly (methylmethacrylate)(PMMA)/poly (styrene-co-acrylonitrile)(SAN) ternary blends. Colloid Polym. Sci. 287(2), 147-155 (2009 Feb)

44. E. Kar, N. Bose, S. Das, N. Mukherjee, S. Mukherjee, Temperature dependent dielectric properties of self-standing and flexible poly (vinylidene fluoride) films infused with $\mathrm{Er} 3+$ doped $\mathrm{GeO} 2$ and $\mathrm{SiO} 2$ nanoparticles. J. Appl. Polym. Sci. (2016). https://doi.org/10.1002/app.44016

Publisher's note Springer Nature remains neutral with regard to jurisdictional claims in published maps and institutional affiliations. 